\title{
ARTICLE \\ Assessment of the Protective Capacity of Vadoze Zone over Aquifer Systems Using Secondary Geoelectrical Parameters: A Case Study of Kaltungo Area North East, Nigeria
}

\author{
E.Y. Mbiimbe ${ }^{1 *}$ I.B. Goni ${ }^{2}$ J.M. El-Nafaty ${ }^{2}$ A.U. Yuguda ${ }^{1}$ \\ 1. Department of Geology, Gombe State University \\ 2. Department of Geology, University of Maiduguri
}

\begin{tabular}{l} 
ARTICLE INFO \\
Article history \\
Received: 7 January 2020 \\
Accepted: 14 January 2020 \\
Published Online: 31 August 2020 \\
\hline Keywords: \\
Assessment \\
Protective Capacity \\
Vadose zone \\
Aquifer systems \\
Secondary Geoelectric Parameters and \\
Kaltungo Area
\end{tabular}

\begin{abstract}
An assessment of the protective capacity of the vadose zone overlying the aquifer systems in the Kaltungo area was carried out to determine its influence on groundwater quality. Applying the schlumberger array with a maximum electrode spread $\mathrm{AB} / 2=100 \mathrm{~m}$ through VES, thirty water well points were surveyed using Omega terrameter (PIOSO1) resistivity meter. The field data was first subjected to manual interpretation through curve marching and then digitized modeled curves using computer software. The interpreted data revealed that the area is characterized by eleven different curve types representing three to five geo electrical layers. In order to assess the protective capacity of the vadoze zone over the aquifer systems, the longitudinal conductance (S) and transverse resistance (T) (secondary geoelectric parameters) were computed from the primary data using the Dar Zarouk formula. The values of S obtained range from 0.0018 to 0.4056 ohms with a mean value of $0.0135 \mathrm{ohms}$ while the values of $\mathrm{T}$ range from $0.55 \mathrm{ohms}$ to $1195.68 \mathrm{ohms}$ with a mean value of $39.84 \mathrm{ohms}$. The values of S and T obtained reveal that $90 \%$ of probed points has poor protective capacity, $10 \%$ has moderate protective capacity and $83 \%$ has high transmissivity, $17 \%$ has intermediate transmissivity. The $\mathrm{T}$ and $\mathrm{S}$ values are skewed towards poorly protective capacity thus making groundwater in the area highly vulnerable to contamination from the surface. To achieve good groundwater quality in the area, proper completion of newly constructed wells should install protective casing through the entire vadose zone.
\end{abstract}

\section{Introduction}

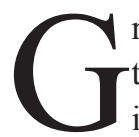
roundwater remains the most readily available alternative source of supply to humanity especially in areas with limited access to surface water. Aquifer systems in geological complex terrain like crystalline basement vary in nature and extent depending on the bed rock and its degree of weathering. The quality of water from such aquifer systems is determined by a number of factors such as the thickness and composition of the materials in the overburden (vadose zone), the depth of occurrence and the nature of human activities in the area. An effective groundwater protection is given by protective layers of the vadose zone with sufficient thickness and

*Corresponding Author:

E.Y. Mbiimbe,

Department of Geology, Gombe State University;

Email: edwinyenika2016@gmail.com 
low hydraulic conductivity ${ }^{[18,26]}$.

Surface geophysical measurements provide an alternative approach for estimation of some of the aquifer properties ${ }^{[2]}$. In the past 3 decades severl investigators have studied the relations between aquifer parameters and geoelectric properties ${ }^{[4,10,13,16,19,23,24]}$.

In this study 30 VES were conducted at preselected stations employing Schlumberger array. The points were selected based on their proximity to existing production wells with the aim of assessing the protective capacity of the vadose zone to the underlying aquifers systems. The background to this study was conceived from the fact that variations in resistivity of subsurface materials is due to variation in the geology and their characteristic compositions. Transverse resistance (T) and longitudinal conductance (S) (Dar Zarouk Parameters) of the vadose zone for the study area were computed from measured field resistivity data and used to assess the protective capacity of the vadose zone over the aquifer systems.

\section{Geology of the Study Area}

The study area is part of the Gombe sub-basin of the upper Benue Trough and is geographically located between latitude $9^{0} 45^{1}$ and $9^{0} 50^{1} \mathrm{~N}$, Longitude $11^{0} 15^{11}$ and $11^{0} 20^{1} \mathrm{E}$. The geology of the area is characterized by crystalline basement rocks mainly coarse porphyritic granite, medium grain granite and biotite granite as well as the intrusion of pegmatite and basalt. The sedimentary succession is defined by Cretaceous sediments of the Bima sandstone. (Figure 1). Comprehensive geology of this sub-basin has been discussed in the works of Benkhelil et'al ${ }^{[5]}$, Zaborski et al ${ }^{[27]}$, Mboringong et al ${ }^{[17]}$.

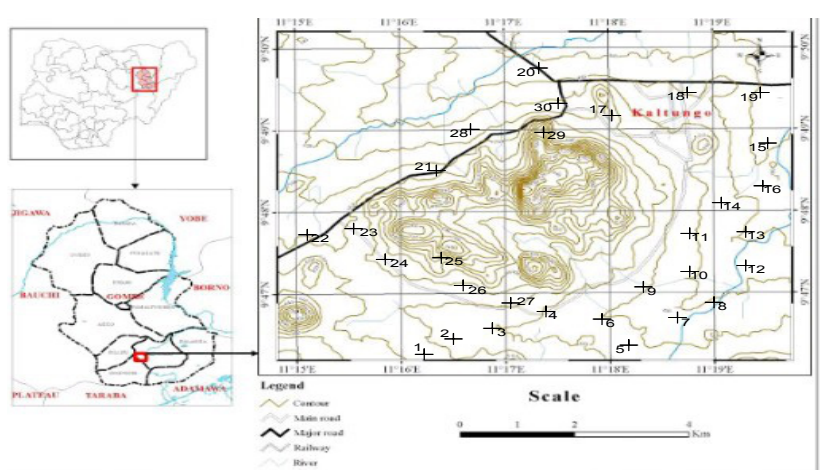

Figure 1. Location map of the study area showing VES points

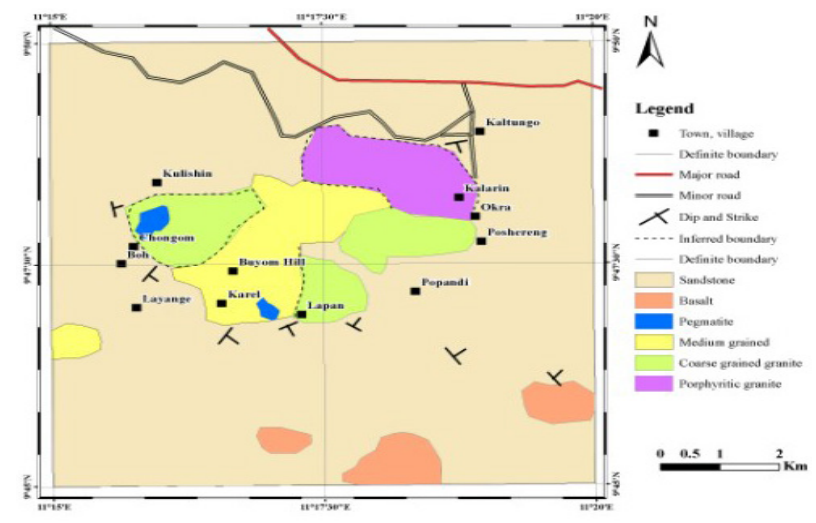

Figure 2. Geologic Map of the study area (Modified from $\mathrm{Sa}$ 'ad and Baba ${ }^{[25]}$ )

\section{Materials and Methods}

\subsection{Theoretical Basis}

An effective groundwater protection is given by protective layers (vadose zone) with sufficient thickness and low hydraulic conductivity leading to longer residence time of percolating water ${ }^{[18]}$. Residence time of percolating water into the aquifer through materials with large pore spaces is shorter than that for smaller pore spaces and as a result water moves faster leading to poor natural filtration process.

\subsection{Data Acquisition and Processing}

The data for this study is of two sets; the field data (primary) and the processed data (secondary geoelectric data). The primary data was generated in the field from investigating thirty probe points using OhmegaTerrameter employing Schlumberger electrode configuration with a maximum spread of $200 \mathrm{~m}$ at $(\mathrm{AB} / 2=100 \mathrm{~m})$. The field data generated in form of apparent resistivity versus electrode spread was interpreted using WINRESIST computer software to give layer resistivity and thickness for each VES point. The interpreted VES results (layer resistivity values and thicknesses) were used to compute the secondary geo-electric parameters, also known as Dar-Zarrouk parameters. These parameters include the Longitudinal Unit Conductance (S) and Transverse Unit Resistance (T).

\subsection{Longitudinal Conductance}

The longitudinal conductance (S) is the geo-electric parameter used to define target areas of groundwater potential.

$\mathrm{S}=\mathrm{h} / \rho \mathrm{a}$

Where $\mathrm{S}$ is the longitudinal conductance, $\mathrm{h}$ is thickness 
and $\rho \mathrm{a}$ is apparent resistivity of the aquiferous layer. For the purpose of this study the resistivity values of the layers overlying the perceived aquiferous zone was used to compute the $S$ values (table 4)

\subsection{Transverse Resistance}

The transverse resistance (T) is one of the parameters used to define target areas of good groundwater potential. It has a direct relation with transmissivity and the highest $\mathrm{T}$ values reflect most likely the highest transmissivity values of the aquifers or aquiferous zones. The transverse resistance (T) is correlated with aquifer transmissivity to establish the functional relationship of the vadose zone and the underlying aquifer in terms of hydraulic communication. This parameter has been used in this study to evaluate the capacity of the top soil (vadose zone) overlying the aquifer system so as to determine its ability to allow infiltrating water to the aquifer. The assumption is that when geologic materials have high transmissivity, the tendency is for them to permit high infiltration into the underlying aquifer systems. The values of $T$ for each VES points were computed using the formula below.

$\mathrm{T}=\mathrm{h} . \rho \mathrm{a}$

Where $\mathrm{T}$ is the transverse resistance, $\mathrm{h}$ is thickness and $\rho$ a is apparent resistivity of the aquiferous layer

\subsection{Vadoze Zone Protective Caoacity}

Vadoze zone protective capacity (VZPC) is the capacity of the overburden unit to impede and filter percolating ground surface polluting liquid into the aquiferous unit. This concept was derived from Henriet's 1976 relationship that "the protective capacity of the overburden (vadose zone) is proportional to its longitudinal conductance $\mathrm{S}$ which in terms of aquifer protection gets a dimension of time (infiltration time)". The second order geo-electric parameter (Dar Zarrouk parameter) was evaluated from the primary/first order parameters (using equation 1) (thickness and resistivity) of the geo-electric subsurface layers which were used in the classification of the protective capacity of the vadoze zone over the aquifer systems of the area. According to Oladapo ${ }^{[20]}$ the protective capacity of the vadose zone over an aquifer can be classified based on total unit conductance $(\Sigma \mathrm{S})$; Excellent $(\mathrm{S}>5)$, very good $(5 \leq S<10)$, good $(0.7 \leq S<5)$, moderate $(0.2 \leq S<0.7)$, weak $(0.1 \leq \mathrm{S}<0.2)$ and poor $(\mathrm{S}<0.1)$.

As the hydraulic conductivity is directly proportional to the resistivity ${ }^{[13]}$ and the product of the resistivity for its thickness, it is defined as being the transverse resistance (T), on a purely empirical basis and it can be admitted that the transmissivity of an aquifer is directly proportional to its transverse resistance ${ }^{[12]}$. Clay layer corresponds to low resistivities and low hydraulic conductivities, and vice versa, hence, the protective capacity of the overburden could be considered as being proportional to the ratio of thickness to resistivity - longitudinal conductance (S). In the present study, layers found above the potential aquifers have generally been considered as the vadose zone and as such their transmissivities (T) have been computed using equation 2 above. Adopting Culled 1982 classification the $\mathrm{T}$ values were categorized as follows: Very high transmissivity magnitude $(T \geq 1000)$, High transmissivity magnitude $(100 \leq \mathrm{T}<1000)$, intermediate transmissivity magnitude $(10 \leq \mathrm{T}<100)$, Low transmissivity magnitude $(1 \leq \mathrm{T}<10)$, very low transmissivity magnitude $(0.1 \leq \mathrm{T}<1)$ and imperceptible transmissivity magnitude $(\mathrm{T}<$ $0.1)$

\section{Result and Discussion}

Table 1. Result of Interpretation of VES Curves from the Study area

\begin{tabular}{|c|c|c|c|c|c|c|}
\hline VES No. & Layers No. & $\mathrm{R}(\Omega)$ & Layer Thickness (m) & Inferred Lithology & Curves Types and $\%$ error & Inferred Aquifer \\
\hline 1 & $\begin{array}{l}1 . \\
2 . \\
3 . \\
4 .\end{array}$ & $\begin{array}{l}150 \\
17.4 \\
7188 \\
31.3\end{array}$ & $\begin{array}{c}2.92 \\
3.25 \\
8 \\
-\end{array}$ & $\begin{array}{c}\text { - Top soil } \\
\text {-Weathered basement } \\
\text { - Fractured basement } \\
\text { Fresh basement }\end{array}$ & HK & Aquifer \\
\hline 2 & $\begin{array}{l}1 . \\
2 . \\
3 . \\
4 .\end{array}$ & $\begin{array}{c}149 \\
110 \\
31.6 \\
21097\end{array}$ & $\begin{array}{c}1.55 \\
10 \\
15.6 \\
-\end{array}$ & $\begin{array}{l}\text { - Top soil } \\
\text {-Weathered basement } \\
\text { - Fractured basement } \\
\text { - Fresh basement }\end{array}$ & $\mathrm{QH}$ & Aquifer \\
\hline 3 & $\begin{array}{l}1 . \\
2 . \\
3 .\end{array}$ & $\begin{array}{l}49.2 \\
376 \\
27.7\end{array}$ & $\begin{array}{c}5.25 \\
13 \\
-\end{array}$ & $\begin{array}{l}\text { - Top soil } \\
\text {-Weathered basement } \\
\text { - Fresh basement }\end{array}$ & $\mathrm{H}$ & Aquifer \\
\hline 4 & $\begin{array}{l}1 . \\
2 . \\
3 . \\
4 .\end{array}$ & $\begin{array}{l}86.1 \\
26.1 \\
104 \\
425\end{array}$ & $\begin{array}{l}1.48 \\
1.23 \\
8.29\end{array}$ & $\begin{array}{l}\text { - Top soil } \\
\text {-Weathered basement } \\
\text { - Fractured basement } \\
\text { - Fresh basement }\end{array}$ & HA & Aquifer \\
\hline
\end{tabular}


Journal of Environmental \& Earth Sciences | Volume 02 | Issue 02 | October 2020

\begin{tabular}{|c|c|c|c|c|c|c|}
\hline 5 & $\begin{array}{l}1 . \\
2 . \\
3 .\end{array}$ & $\begin{array}{l}163 \\
388 \\
90.7\end{array}$ & $\begin{array}{c}3.84 \\
18 \\
-\end{array}$ & $\begin{array}{c}\text { Top soil } \\
\text {-Weathered basement } \\
\text { - Fresh basement }\end{array}$ & $\mathrm{K}$ & Aquifer \\
\hline 6 & $\begin{array}{l}1 . \\
2 . \\
3 . \\
4 .\end{array}$ & $\begin{array}{l}37.3 \\
55.6 \\
237 \\
15.5\end{array}$ & $\begin{array}{c}6.93 \\
5.39 \\
6.68 \\
-\end{array}$ & $\begin{array}{l}\text { - Top soil } \\
\text {-Weathered basement } \\
\text { - Fractured basement } \\
\text { - Fresh basement }\end{array}$ & $\mathrm{AH}$ & Aquifer \\
\hline 7 & $\begin{array}{l}1 . \\
2 . \\
3 . \\
4 .\end{array}$ & $\begin{array}{c}99.1 \\
55.4 \\
16.6 \\
10749\end{array}$ & $\begin{array}{c}5 \\
13 \\
17.4 \\
-\end{array}$ & $\begin{array}{l}\text { - Top soil } \\
\text {-Weathered Basement } \\
\text { - Fractured Basement } \\
\text { - Fresh Basement }\end{array}$ & $\mathrm{QH}$ & Aquifer \\
\hline 8 & $\begin{array}{l}1 . \\
2 . \\
3 . \\
4 .\end{array}$ & $\begin{array}{c}24.8 \\
4.26 \\
3324 \\
37\end{array}$ & $\begin{array}{c}2.06 \\
2.96 \\
5.52 \\
-\end{array}$ & $\begin{array}{l}\text { - Top soil } \\
\text {-Weathered basement } \\
\text { - Fractured basement } \\
\text { - Fresh basement }\end{array}$ & HA & Aquifer \\
\hline 9 & $\begin{array}{l}1 . \\
2 . \\
3 . \\
4 .\end{array}$ & $\begin{array}{c}171 \\
399 \\
153 \\
0.862\end{array}$ & $\begin{array}{l}1.57 \\
0.66 \\
40.7\end{array}$ & $\begin{array}{l}\text { - Top soil } \\
\text {-Weathered basement } \\
\text { - Fractured basement } \\
\text { - Fresh basement }\end{array}$ & $\mathrm{KH}$ & Aquifer \\
\hline 10 & $\begin{array}{l}1 . \\
2 . \\
3 . \\
4 .\end{array}$ & $\begin{array}{c}3.9 \\
54.3 \\
9.44 \\
95.4\end{array}$ & $\begin{array}{c}0.142 \\
3.46 \\
2.63 \\
-\end{array}$ & $\begin{array}{c}\text { - Top soil } \\
\text {-Weathered basement } \\
\text { - Fractured basement } \\
\text { - Fresh basement }\end{array}$ & HK & Aquifer \\
\hline 11 & $\begin{array}{l}1 . \\
2 . \\
3 .\end{array}$ & $\begin{array}{l}80.7 \\
26.1 \\
170\end{array}$ & $\begin{array}{c}5.43 \\
3.58 \\
-\end{array}$ & $\begin{array}{c}\text { Top soil } \\
\text {-Weathered basement } \\
\text { - Fresh basement }\end{array}$ & $\mathrm{H}$ & Aquifer \\
\hline 12 & $\begin{array}{l}1 . \\
2 . \\
3 . \\
4 . \\
5 .\end{array}$ & $\begin{array}{c}251 \\
116 \\
369 \\
18.5 \\
14180\end{array}$ & $\begin{array}{c}2.46 \\
1.93 \\
4.29 \\
9.46 \\
-\end{array}$ & $\begin{array}{c}\text { - Top soil } \\
\text { - Slightly Weathered basement } \\
\text {-Weathered basement } \\
\text { - Fractured basement } \\
\text { - Fresh basement }\end{array}$ & HKH & Aquifer \\
\hline 13 & $\begin{array}{l}1 . \\
2 . \\
3 . \\
4 .\end{array}$ & $\begin{array}{l}89.8 \\
220 \\
14.8 \\
444\end{array}$ & $\begin{array}{c}2.81 \\
5.63 \\
8.6 \\
-\end{array}$ & $\begin{array}{l}\text { - Top soil } \\
\text {-Weathered basement } \\
\text { - Fractured basement } \\
\text { - Fresh basement }\end{array}$ & $\mathrm{KH}$ & Aquifer \\
\hline 14 & $\begin{array}{l}1 . \\
2 . \\
3 . \\
4 .\end{array}$ & $\begin{array}{l}53.8 \\
147 \\
561 \\
94.5\end{array}$ & $\begin{array}{c}0.323 \\
9.99 \\
9.03 \\
-\end{array}$ & $\begin{array}{c}\text { - Top soil } \\
\text {-Weathered basement } \\
\text { - Fractured basement } \\
\text { - Fresh basement }\end{array}$ & HK & Aquifer \\
\hline 15 & $\begin{array}{l}1 . \\
2 . \\
3 . \\
4 . \\
5 .\end{array}$ & $\begin{array}{l}39.3 \\
62.3 \\
23.5 \\
512 \\
33.9 \\
\end{array}$ & $\begin{array}{c}0.9 \\
1.02 \\
2.18 \\
4.66 \\
- \\
\end{array}$ & $\begin{array}{c}\text { - Top soil } \\
\text { - Slightly Weathered basement } \\
\text {-Weathered basement } \\
\text { - Fractured basement } \\
\text { - Fresh basement }\end{array}$ & KHK & Aquifer \\
\hline 16 & $\begin{array}{l}1 . \\
2 . \\
3 . \\
4 . \\
5 .\end{array}$ & $\begin{array}{c}28.1 \\
362 \\
28.3 \\
119 \\
0.434 \\
\end{array}$ & $\begin{array}{c}1.64 \\
1.67 \\
5.86 \\
15.5 \\
- \\
\end{array}$ & $\begin{array}{c}\text { - Top soil } \\
\text { - Slightly Weathered basement } \\
\text {-Weathered basement } \\
\text { Fractured basement } \\
\text { Fresh basement }\end{array}$ & KHK & Aquifer \\
\hline 17 & $\begin{array}{l}1 . \\
2 . \\
3 .\end{array}$ & $\begin{array}{c}62.7 \\
10.3 \\
11095\end{array}$ & $\begin{array}{c}9.78 \\
13.4 \\
-\end{array}$ & $\begin{array}{c}\text { - Top soil } \\
\text {-Weathered basement } \\
\text { - Fresh basement }\end{array}$ & $\mathrm{H}$ & Aquifer \\
\hline 18 & $\begin{array}{l}1 . \\
2 . \\
3 . \\
4 .\end{array}$ & $\begin{array}{l}236 \\
113 \\
9.33 \\
104\end{array}$ & $\begin{array}{c}0.783 \\
3.38 \\
1.52 \\
-\end{array}$ & $\begin{array}{l}\text { - Top soil } \\
\text {-Weathered basement } \\
\text { - Fractured basement } \\
\text { - Fresh basement }\end{array}$ & HK & Aquifer \\
\hline 19 & $\begin{array}{l}1 . \\
2 . \\
3 . \\
4 .\end{array}$ & $\begin{array}{c}114 \\
29.2 \\
11.5 \\
3307\end{array}$ & $\begin{array}{c}0.941 \\
11.6 \\
15.6 \\
-\end{array}$ & $\begin{array}{c}\text { - Top soil } \\
\text {-Weathered basement } \\
\text { - Fractured basement } \\
\text { - Fresh basement }\end{array}$ & $\mathrm{QH}$ & Aquifer \\
\hline
\end{tabular}




\begin{tabular}{|c|c|c|c|c|c|c|}
\hline 20 & $\begin{array}{l}1 . \\
2 . \\
3 . \\
4 . \\
5 .\end{array}$ & $\begin{array}{c}243 \\
105 \\
27.1 \\
119 \\
6491\end{array}$ & $\begin{array}{c}0.51 \\
4.53 \\
4.43 \\
36.6 \\
-\end{array}$ & $\begin{array}{l}\text { - Top soil } \\
\text { - Slightly Weathered basement } \\
\text {-Weathered basement } \\
\text { - Fractured basement } \\
\text { - Fresh basement }\end{array}$ & QHA & Aquifer \\
\hline 21 & $\begin{array}{l}1 . \\
2 . \\
3 . \\
4 .\end{array}$ & $\begin{array}{c}631.1 \\
81.3 \\
166.0 \\
147.9\end{array}$ & $\begin{array}{c}1.1 \\
14.3 \\
70.8 \\
-\end{array}$ & $\begin{array}{l}\text { - Top soil } \\
\text { - Weathered basement } \\
\text { - Fractured basement } \\
\text { - Fresh basement }\end{array}$ & HQ & Aquifer \\
\hline 22 & $\begin{array}{l}1 . \\
2 . \\
3 .\end{array}$ & $\begin{array}{c}275.7 \\
103.0 \\
2546.6\end{array}$ & $\begin{array}{c}0.8 \\
24.3 \\
-\end{array}$ & $\begin{array}{l}\text { - Top soil } \\
\text { - Weathered basement } \\
\text { - Fresh basement }\end{array}$ & $\mathrm{H}$ & Aquifer \\
\hline 23 & $\begin{array}{l}1 . \\
2 . \\
3 .\end{array}$ & $\begin{array}{c}403.5 \\
66.3 \\
726.1\end{array}$ & $\begin{array}{c}1.8 \\
14.6 \\
-\end{array}$ & $\begin{array}{l}\text { - Top soil } \\
\text { - Weathered basement } \\
\text { - Fresh basement }\end{array}$ & $\mathrm{H}$ & Aquifer \\
\hline 24 & $\begin{array}{l}1 . \\
2 . \\
3 . \\
4 .\end{array}$ & $\begin{array}{c}380.5 \\
60.3 \\
1069.9 \\
789.2\end{array}$ & $\begin{array}{c}0.8 \\
3.2 \\
56.4 \\
-\end{array}$ & $\begin{array}{l}\text { - Top soil } \\
\text { - Weathered basement } \\
\text { - Fractured basement } \\
\text { - Fresh basement }\end{array}$ & HQ & Aquifer \\
\hline 25 & $\begin{array}{l}1 . \\
2 . \\
3 .\end{array}$ & $\begin{array}{c}112.8 \\
675.2 \\
77.8\end{array}$ & $\begin{array}{c}10.6 \\
56.2 \\
-\end{array}$ & $\begin{array}{l}\text { - Top soil } \\
\text { - Weathered basement } \\
\text { - Fresh basement }\end{array}$ & K & Aquifer \\
\hline 26 & $\begin{array}{l}1 . \\
2 . \\
3 .\end{array}$ & $\begin{array}{c}200.6 \\
22.6 \\
1003.8 \\
\end{array}$ & $\begin{array}{c}1.6 \\
4.8 \\
- \\
\end{array}$ & $\begin{array}{c}\text { - Top soil } \\
\text { - Weathered basement } \\
\text { - Fresh basement }\end{array}$ & $\mathrm{H}$ & Aquifer \\
\hline 27 & $\begin{array}{l}1 . \\
2 . \\
3 . \\
4 .\end{array}$ & $\begin{array}{c}231.0 \\
28.7 \\
249.5 \\
327.0\end{array}$ & $\begin{array}{c}3.0 \\
10.1 \\
49.9 \\
-\end{array}$ & $\begin{array}{l}\text { - Top soil } \\
\text { - Weathered basement } \\
\text { - Fractured basement } \\
\text { - Fresh basement }\end{array}$ & HK & Aquifer \\
\hline 28 & $\begin{array}{l}1 . \\
2 . \\
3 . \\
4 . \\
5 .\end{array}$ & $\begin{array}{c}195.3 \\
24.2 \\
401.9 \\
23.0 \\
32.8 \\
\end{array}$ & $\begin{array}{c}1.8 \\
3.0 \\
11.0 \\
39.5 \\
-\end{array}$ & $\begin{array}{l}\text { - Top soil } \\
\text { - Slightly weathered } \\
\text { - Weathered basement } \\
\text { - Fractured basement } \\
\text { - Fresh basement }\end{array}$ & HKH & Aquifer \\
\hline 29 & $\begin{array}{l}1 . \\
2 . \\
3 . \\
4 .\end{array}$ & $\begin{array}{c}423.1 \\
91.0 \\
212.6 \\
942.0\end{array}$ & $\begin{array}{c}1.1 \\
5.6 \\
28.7 \\
-\end{array}$ & $\begin{array}{l}\text { - Top soil } \\
\text { - Weathered basement } \\
\text { - Fractured basement } \\
\text { - Fresh basement }\end{array}$ & HK & Aquifer \\
\hline 30 & $\begin{array}{l}1 . \\
2 . \\
3 .\end{array}$ & $\begin{array}{c}435.7 \\
89.4 \\
3020.6 \\
\end{array}$ & $\begin{array}{c}0.8 \\
14.2 \\
-\end{array}$ & $\begin{array}{c}\text { - Top soil } \\
\text { - Weathered basement } \\
\text { - Fresh basement }\end{array}$ & $\mathrm{H}$ & Aquifer \\
\hline
\end{tabular}

\subsection{Geo-Electric Section}

The data analysis from the study area shows a three to five layers geo-electric succession (Figure 3 and 4). This succession comprises of the dry topsoil, slightly weathered, weathered basement, fractured basement and Fresh basement. Weathered and fractured zones represented by low and fairly high resistivity units, respectively, are considered to be the potential groundwater bearing zones. Dike el al. ${ }^{[8]}$
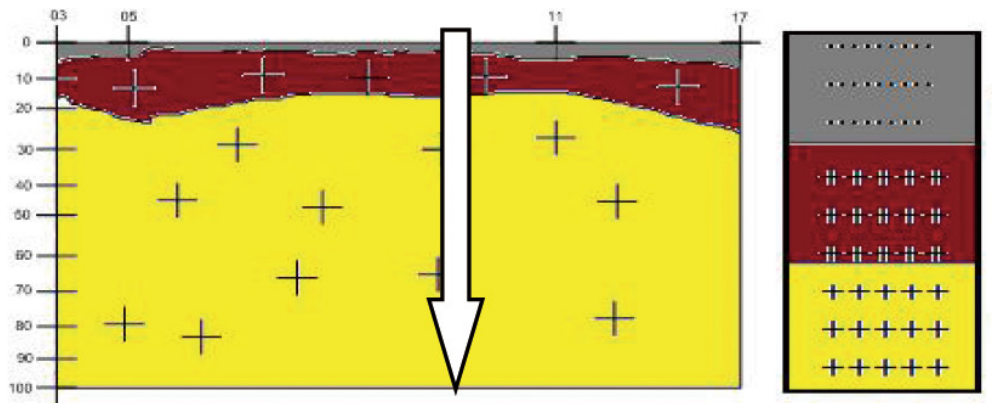

Top soil

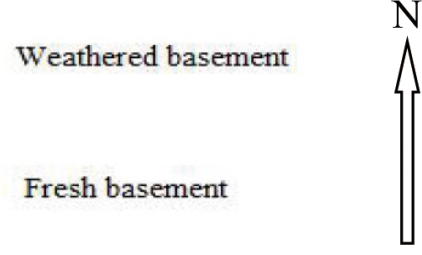

Figure 3. Geo electric Section of Ves 03, 05, 11 and 17 (3 layers) 


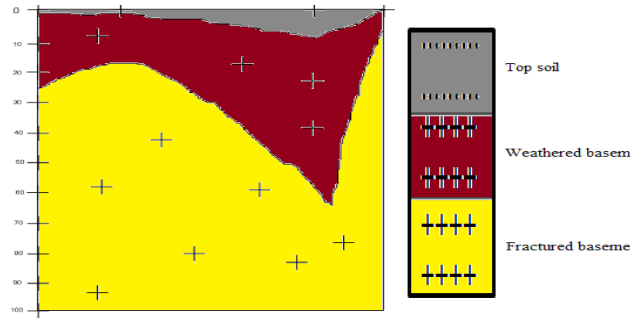

Figure 4. Geo electric Section of Ves 22, 2325 and 26 (3 layers)

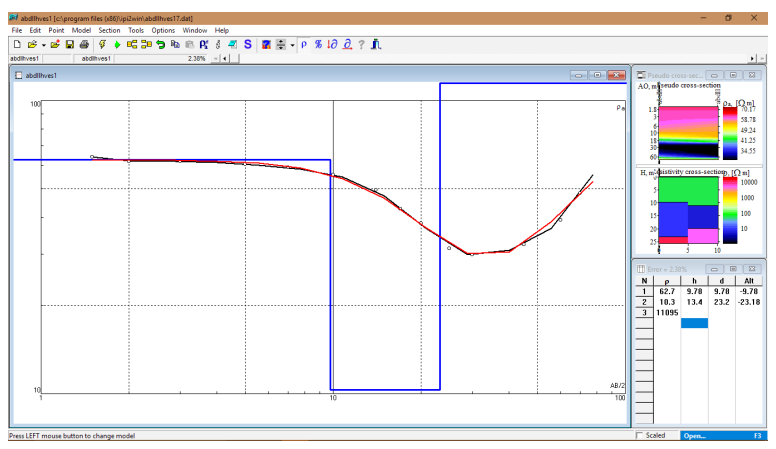

Figure 5. A three layer type curve

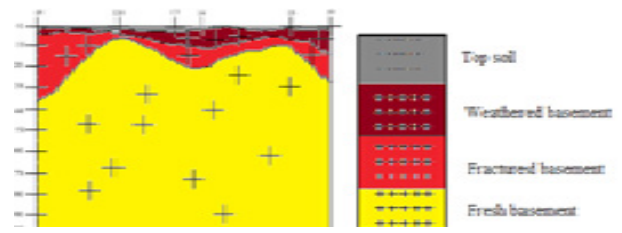

Figure 6. Geo electric Section of Ves 1, 2, 4, 6, 7 and 8 (4 layers)

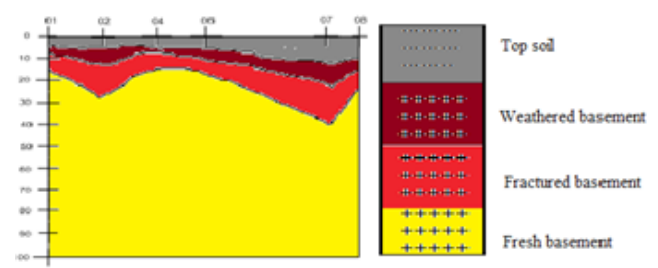

Figure 7. Geo electric Section of Ves 9, 10, 13, 14, 18 and 19 (4 layers)

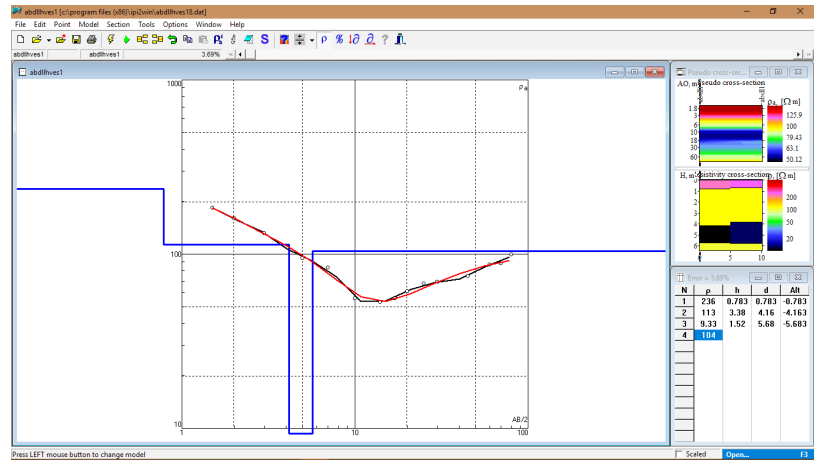

Figure 8. A four layer type curve
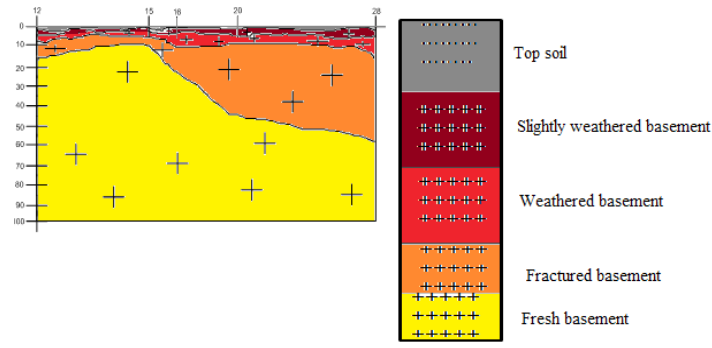

Figure 9. Geo electric Section of Ves 12, 15, 16, 20 and 28 (5 layers)

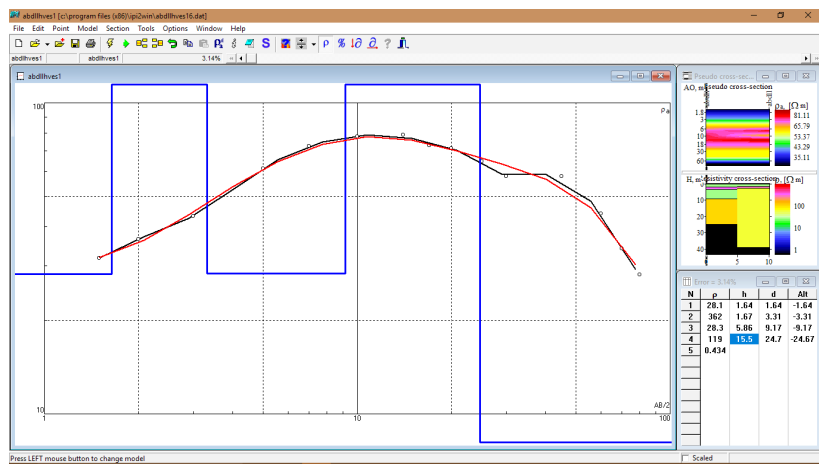

Figure 10. A five layer Type Curve

Table 2. Evaluation of the Longitudinal Conductance and Transverse Resistance of the Layers Obtained from each VES Location

\begin{tabular}{|c|c|c|c|c|c|c|c|c|}
\hline VES NO & $\mathbf{S 1} \boldsymbol{\Omega}$ & $\mathbf{S 2} \boldsymbol{\Omega}$ & $\mathbf{S 3} \boldsymbol{\Omega}$ & $\mathbf{S 4} \boldsymbol{\Omega}$ & $\mathbf{T 1} \boldsymbol{\Omega}$ & $\mathbf{T 2} \boldsymbol{\mathbf { S }}$ & $\mathbf{T 3} \boldsymbol{\Omega}$ & $\mathbf{T} \mathbf{\Omega}$ \\
\hline $\mathbf{1}$ & 0.0197 & 0.2443 & 0.0011 & & 444.00 & 73.95 & 57504.00 & \\
\hline $\mathbf{2}$ & 0.0104 & 0.0909 & 0.4937 & & 230.95 & 1100.00 & 492.96 & \\
\hline $\mathbf{3}$ & 0.1067 & 0.0346 & & & 258.30 & 4888.00 & & \\
\hline $\mathbf{4}$ & 0.0172 & 0.0457 & 0.0797 & & 127.43 & 33.09 & 862.16 & \\
\hline $\mathbf{5}$ & 0.0236 & 0.0464 & & & 625.92 & 6984.00 & & \\
\hline $\mathbf{6}$ & 0.1859 & 0.0969 & 0.0282 & & 258.64 & 299.68 & 1583.16 & \\
\hline $\mathbf{7}$ & 0.0505 & 0.2347 & 1.0482 & & 495.50 & 720.20 & 288.84 & \\
\hline $\mathbf{8}$ & 0.0831 & 0.6854 & 0.0017 & & 51.09 & 12.44 & 188348.48 & \\
\hline $\mathbf{9}$ & 0.0092 & 0.0017 & 0.2660 & & 268.47 & 263.34 & 6227.10 & \\
\hline $\mathbf{1 0}$ & 0.0364 & 0.0637 & 0.2786 & & 0.55 & 187.88 & 24.83 & \\
\hline
\end{tabular}


Journal of Environmental \& Earth Sciences | Volume 02 | Issue 02 | October 2020

\begin{tabular}{|c|c|c|c|c|c|c|c|c|}
\hline 11 & 0.0673 & 0.1372 & & & 438.20 & 93.44 & & \\
\hline 12 & 0.0098 & 0.0166 & 0.0166 & 0.5114 & 617.46 & 223.88 & 1583.01 & 175.01 \\
\hline 13 & 0.0313 & 0.0256 & 0.5838 & & 252.34 & 1238.60 & 127.87 & \\
\hline 14 & 0.0060 & 0.0680 & 0.0161 & & 17.38 & 1468.53 & 5065.83 & \\
\hline 15 & 0.0229 & 0.0164 & 0.0928 & 0.0091 & 35.37 & 63.55 & 51.23 & 2385.92 \\
\hline 16 & 0.584 & 0.0046 & 0.2071 & 0.1303 & 46.08 & 604.54 & 165.84 & 1844.50 \\
\hline 17 & 0.1559 & 1.3009 & & & 613.21 & 139.36 & & \\
\hline 18 & 0.0033 & 0.0299 & 0.1629 & & 184.79 & 381.94 & 14.18 & \\
\hline 19 & 0.0083 & 0.3973 & 1.3565 & & 107.27 & 338.72 & 179.40 & \\
\hline 20 & 0.0021 & 0.0431 & 0.1635 & 0.3076 & 123.93 & 475.65 & 120.05 & 4355.40 \\
\hline 21 & 0.0017 & 0.1759 & 0.4265 & & 694.21 & 1162.59 & 11752.8 & \\
\hline 22 & 0.0029 & 0.2559 & & & 220.56 & 2502.9 & & \\
\hline 23 & 0.0045 & 0.2202 & & & 726.30 & 967.98 & & \\
\hline 24 & 0.0021 & 0.0531 & 0.0527 & & 304.4 & 192.96 & 60342.36 & \\
\hline 25 & 0.0939 & 0.0531 & & & 1195.68 & 37946.24 & & \\
\hline 26 & 0.0079 & 0.2124 & & & 320.96 & 108.48 & & \\
\hline 27 & 0.0129 & 0.3519 & 299.4 & & 693.0 & 289.87 & 12450.05 & \\
\hline 28 & 0.0092 & 0.1239 & 0.0274 & 1.7174 & 351.54 & 72.6 & 4420.9 & 908.5 \\
\hline 29 & 0.0026 & 0.0615 & 0.1349 & & 465.41 & 509.6 & 6101.62 & \\
\hline 30 & 0.0018 & 0.1588 & & & 348.56 & 1269.48 & & \\
\hline
\end{tabular}

Table 3. Evaluation of the Total Longitudinal Conductance, Total Transverse Resistance and Average Longitudinal Conductance and Average Transverse Resistance each VES Location

\begin{tabular}{|c|c|c|c|c|}
\hline VES NO & $\mathrm{S}$ & PL & $\mathrm{T}$ & $\mathrm{Pt}$ \\
\hline 1 & 0.2651 & 57.37 & 58021.95 & 3814.72 \\
\hline 2 & 0.5950 & 45.78 & 1823.91 & 67.18 \\
\hline 3 & 0.1413 & 129.16 & 5146.30 & 281.99 \\
\hline 4 & 0.1426 & 77.14 & 1022.68 & 92.97 \\
\hline 5 & 0.07 & 312 & 7609.92 & 348.44 \\
\hline 6 & 0.1501 & 0.06 & 2141.48 & 112.71 \\
\hline 7 & 1.3334 & 26.55 & 1504.54 & 42.50 \\
\hline 8 & 0.7702 & 0.0031 & 18412.01 & 1753.52 \\
\hline 9 & 0.2769 & 155.04 & 6758.91 & 157.44 \\
\hline 10 & 0.3787 & 16.46 & 213.26 & 34.21 \\
\hline 11 & 0.2045 & 44.06 & 531.64 & 59.01 \\
\hline 12 & 0.5494 & 33.02 & 2599.36 & 143.29 \\
\hline 13 & 0.6407 & 26.66 & 1618.81 & 94.78 \\
\hline 14 & 0.0901 & 95.95 & 6551.74 & 355.40 \\
\hline 15 & 0.1412 & 62.04 & 2536.07 & 289.51 \\
\hline 16 & 0.4004 & 61.61 & 2659.96 & 107.82 \\
\hline 17 & 1.4568 & 15.91 & 752.57 & 32.47 \\
\hline 18 & 0.1961 & 28.98 & 544.91 & 95.88 \\
\hline 19 & 1.7621 & 15.97 & 625.39 & 22.22 \\
\hline 20 & 0.5163 & 89.23 & 5075.03 & 110.16 \\
\hline 21 & 0.6040 & 13609.6 & 142.69 & 157.88 \\
\hline 22 & 0.2388 & 2723.46 & 15.11 & 108.50 \\
\hline 23 & 0.2247 & 1694.28 & 72.99 & 103.31 \\
\hline 24 & 0.1079 & 60839.72 & 559.78 & 1007.28 \\
\hline 25 & 0.1515 & 39141.92 & 436.96 & 519.27 \\
\hline 26 & 0.2203 & 429.44 & 29.05 & 67.10 \\
\hline 27 & 299.7648 & 13432.92 & 0.21 & 216.31 \\
\hline 28 & 1.8779 & 5681.01 & 29.45 & 102.73 \\
\hline 29 & 0.199 & 7076.62 & 177.89 & 0.01 \\
\hline 30 & 0.1606 & 1618.04 & 93.40 & 107.87 \\
\hline
\end{tabular}


3.2 Protective Capacity Evaluation of the Vadoze Zone

The nature of the materials that overlain the mapped aquifers were evaluated using the layer parameters (i.e. resistivity and thickness), to determine its capacity to prevent infiltration of unwanted fluids into the aquifer. It should be noted that the earth materials act as natural filter to percolating fluids; therefore its ability to retard and filter percolating ground surface polluting fluids is a measure of its protective capacity ${ }^{[22]}$. That is to say that the geologic materials overlying an aquifer could act as seal in preventing the fluid from percolating into it.

The longitudinal unit conductance (S) values of the overburden materials obtained from the study area, ranges from 0.0018 to 0.4056 ohms (Table 4) with a mean value of $0.0135 \mathrm{ohms}$. Clayey overburden, which is characterized by relatively high longitudinal conductance, offers protection to the underlying aquifer. According to the classification of Oladapo and Akintorinwa ${ }^{[21]}$, the longitudinal unit conductance $(\mathrm{S})$ values from the study area enabled us to classify the area into poor, $(\mathrm{S}<0.1)$, weak, $(0.1 \leq \mathrm{S}<0.2)$, moderate $(0.2 \leq \mathrm{S}<0.7)$ and good, $(0.7 \leq \mathrm{S}<5)$, very good $(5 \leq \mathrm{S}<10)$ and excellent $(\mathrm{S}>10)$ protective capacity zones. Where the conductance is greater than 10 mhos are considered zones of excellent protective capacity. This study has revealed that the overburden materials (vadose zone) in the VES 01, 04, 05, 08, 09,11, 12, 13, 14, $15,16,18,20,22,23,24,25,26,27,29$ and 30 have poor protective capacity while VES $02,03,06,10,17,21$ and 28 are characterized by weak protective capacity. Furthermore, the VES 07, 19 and 27 are found to have a moderate protective capacity (Table 4, Figure 10). The result revealed that $90 \%$ of VES within the study area have poor protective capacity, while $10 \%$ have weak to moderate protective capacity.

Table 4. Summary of the vadoze zone protective capacity over the aquifer systems of the study area

\begin{tabular}{|c|c|c|c|c|}
\hline VES No & No. of Overburden layers & Lithology & $\begin{array}{l}\text { Longitudinal Conductance } \\
\left(\sum \mathbf{S}\right)\end{array}$ & Protective Capacity \\
\hline 1 & 1. & - Top soil & 0.02 & $(\mathrm{~S}<0.1)$, (Poor) \\
\hline 2 & $\begin{array}{l}1 . \\
2 .\end{array}$ & $\begin{array}{c}\text { - Top soil } \\
\text { - Weathered basement }\end{array}$ & 0.10 & $(0.1 \leq \mathrm{S}<0.2)$ (Weak) \\
\hline 3 & 1. & - Top soil & 0.11 & $(0.1 \leq \mathrm{S}<0.2)($ Weak $)$ \\
\hline 4 & $\begin{array}{l}1 . \\
2 .\end{array}$ & $\begin{array}{c}\text { - Top soil } \\
\text { - Weathered basement }\end{array}$ & 0.06 & $(\mathrm{~S}<0.1)$, (Poor) \\
\hline 5 & 1. & - Top soil & 0.02 & $(\mathrm{~S}<0.1)$, (Poor) \\
\hline 6 & 1. & - Top soil & 0.19 & $(0.1 \leq \mathrm{S}<0.2)($ Weak $)$ \\
\hline 7 & $\begin{array}{l}1 . \\
2 .\end{array}$ & $\begin{array}{c}\text { - Top soil } \\
\text { - Weathered basement }\end{array}$ & 0.03 & $(\mathrm{~S}<0.1)$, (Poor) \\
\hline 8 & 1. & - Top soil & 0.08 & $(\mathrm{~S}<0.1)$, (Poor) \\
\hline 9 & $\begin{array}{l}1 . \\
2 .\end{array}$ & $\begin{array}{l}\text { - Top soil } \\
\text { - Weathered basement }\end{array}$ & 0.01 & $(\mathrm{~S}<0.1)$, (Poor) \\
\hline 10 & $\begin{array}{l}1 . \\
2 .\end{array}$ & $\begin{array}{c}\text { - Top soil } \\
\text { - Weathered basement }\end{array}$ & 0.1 & $(0.1 \leq \mathrm{S}<0.2)($ Weak $)$ \\
\hline 11 & 1. & - Top soil & 0.07 & $(\mathrm{~S}<0.1)$, (Poor) \\
\hline 12 & $\begin{array}{l}1 . \\
2 . \\
3 .\end{array}$ & $\begin{array}{c}\text { - Top soil } \\
\text { - Slightly weathered } \\
\text { - Weathered basement }\end{array}$ & 0.04 & $(\mathrm{~S}<0.1)$, (Poor) \\
\hline 13 & $\begin{array}{l}1 . \\
2 .\end{array}$ & $\begin{array}{l}\text { - Top soil } \\
\text { - Weathered basement }\end{array}$ & 0.06 & $(\mathrm{~S}<0.1)$, (Poor) \\
\hline 14 & 1. & - Top soil & 0.006 & $(\mathrm{~S}<0.1)$, (Poor) \\
\hline 15 & $\begin{array}{l}1 . \\
2 .\end{array}$ & $\begin{array}{c}\text { - Top soil } \\
\text { - Weathered basement }\end{array}$ & 0.04 & $(\mathrm{~S}<0.1)$, (Poor) \\
\hline 16 & $\begin{array}{l}1 . \\
2 .\end{array}$ & $\begin{array}{l}\text { - Top soil } \\
\text { - Weathered basement }\end{array}$ & 0.06 & $(\mathrm{~S}<0.1)$, (Poor) \\
\hline 17 & 1. & - Top soil & 0.16 & $(0.1 \leq \mathrm{S}<0.2)($ Weak $)$ \\
\hline 18 & $\begin{array}{l}1 . \\
2 .\end{array}$ & $\begin{array}{c}\text { - Top soil } \\
\text { - Weathered basement }\end{array}$ & 0.03 & $(\mathrm{~S}<0.1)$, (Poor) \\
\hline
\end{tabular}


Journal of Environmental \& Earth Sciences | Volume 02 | Issue 02 | October 2020

\begin{tabular}{|c|c|c|c|c|}
\hline 19 & $\begin{array}{l}1 . \\
2 .\end{array}$ & $\begin{array}{c}\text { - Top soil } \\
\text { - Weathered basement }\end{array}$ & 0.4 & $(0.2 \leq \mathrm{S}<0.7)$, (Moderate) \\
\hline 20 & $\begin{array}{l}1 . \\
2 .\end{array}$ & $\begin{array}{c}\text { - Top soil } \\
\text { - Weathered basement }\end{array}$ & 0.05 & $(\mathrm{~S}<0.1)$, (Poor) \\
\hline 21 & $\begin{array}{l}1 . \\
2 .\end{array}$ & $\begin{array}{c}\text { - Top soil } \\
\text { - Weathered basement }\end{array}$ & 0.28 & $(0.1 \leq \mathrm{S}<0.2)$ (Weak) \\
\hline 22 & 1. & - Top soil & 0.003 & $(\mathrm{~S}<0.1)$, , (Poor) \\
\hline 23 & 1. & - Top soil & 0.005 & $(\mathrm{~S}<0.1)$, (Poor) \\
\hline 24 & $\begin{array}{l}1 . \\
2 .\end{array}$ & $\begin{array}{c}\text { - Top soil } \\
\text { - Weathered basement }\end{array}$ & 0.06 & $(\mathrm{~S}<0.1)$, (Poor) \\
\hline 25 & 1. & - Top soil & 0.09 & $(\mathrm{~S}<0.1)$, (Poor) \\
\hline 26 & 1. & - Top soil & 0.01 & $(\mathrm{~S}<0.1)$, (Poor) \\
\hline 27 & $\begin{array}{l}1 . \\
2 .\end{array}$ & $\begin{array}{c}\text { - Top soil } \\
\text { - Weathered basement }\end{array}$ & 0.36 & $(0.2 \leq \mathrm{S}<0.7)$ (Moderate) \\
\hline 28 & $\begin{array}{l}1 . \\
2 . \\
3 .\end{array}$ & $\begin{array}{c}\text { - Top soil } \\
\text { - Slightly weathered } \\
\text { - Weathered basement }\end{array}$ & 0.16 & $(0.1 \leq \mathrm{S}<0.2)($ Weak $)$ \\
\hline 29 & $\begin{array}{l}1 . \\
2 .\end{array}$ & $\begin{array}{c}\text { - Top soil } \\
\text { - Weathered basement }\end{array}$ & 0.06 & $(\mathrm{~S}<0.1)$, (Poor) \\
\hline 30 & 1. & - Top Soil & 0.002 & $(\mathrm{~S}<0.1)$ (Poor) \\
\hline
\end{tabular}

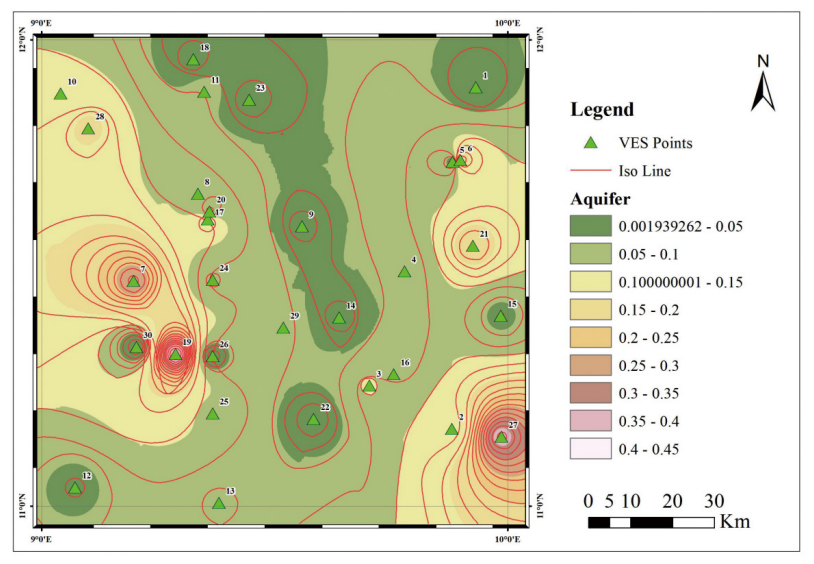

Legend:

$(0.2 \leq \mathrm{S}<0.7)$ Moderate Protective Capacity

$(0.1 \leq \mathrm{S}<0.2)$ Weak Protective Capacity

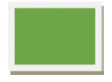

\section{$(\mathbf{S}<0.1)$ (Poor) Poor Protective Capacity}

Figure 11. Vadoze zone protective capacity Map

\subsection{Vadose Zone Transmissivity}

The transmissivity values of the Vadoze zone were evaluated using a relationship between transverse resistance and aquifer transmissivity. The transmissivity values obtained within the area of study range from 0.55 to 1195.68 $\Omega^{2}$ with a mean value of 72.47 . The results as presented in (Table 5) and figure 11 show that the vadose zone in the study area majorly offers less protection to the underlying aquifer systems. Generally from the analysis it shows that $80 \%$ of the points investigated have High, $3.3 \%$ Very High, $13.3 \%$ intermediate and 3.3\% very low Transmissivity magnitude. The points with high to very high values of $\mathrm{T}$ also corresponded with those with poor to weak protective capacity as represented by table 4 and figure 9. These two parameters have thus revealed that the underlying aquifer systems are highly vulnerable to any contaminants emanating from surface activities.

Table 5. Vadoze zone Transmissivity in relation to Transverse Resistance within the VES locations

\begin{tabular}{|c|c|c|c|c|}
\hline $\begin{array}{c}\text { VES } \\
\text { NO }\end{array}$ & $\begin{array}{c}\text { Pa of the } \\
\text { vadoze zone }\end{array}$ & Thickness & Transmissivity & Comment \\
\hline 1 & 150 & 2.96 & 444.00 & High \\
\hline 2 & 149 & 1.55 & 230.95 & High \\
\hline 3 & 49.2 & 5.25 & 258.30 & High \\
\hline 4 & 86.1 & 1.48 & 127.43 & High \\
\hline 5 & 163 & 3.84 & 625.92 & High \\
\hline 6 & 37.3 & 6.93 & 258.64 & High \\
\hline 7 & 99.1 & 5 & 495.50 & High \\
\hline 8 & 24.8 & 2.06 & 51.09 & Intermediate \\
\hline
\end{tabular}




\begin{tabular}{|c|c|c|c|c|}
\hline 9 & 171 & 1.57 & 268.47 & High \\
\hline 10 & 3.9 & 0.142 & 0.55 & Very Low \\
\hline 11 & 80.7 & 5.43 & 438.20 & High \\
\hline 12 & 251 & 2.46 & 617.46 & High \\
\hline 13 & 89.8 & 2.81 & 252.34 & High \\
\hline 14 & 53.8 & 0.323 & 17.38 & Intermediate \\
\hline 15 & 39.3 & 0.9 & 35.37 & Intermediate \\
\hline 16 & 28.1 & 1.64 & 46.08 & Intermediate \\
\hline 17 & 62.7 & 9.78 & 613.21 & High \\
\hline 18 & 236 & 0.78 & 184.79 & High \\
\hline 19 & 114 & 0.94 & 107.27 & High \\
\hline 20 & 243 & 0.51 & 123.93 & High \\
\hline 21 & 631.1 & 1.1 & 694.21 & High \\
\hline 22 & 275.7 & 0.8 & 220.56 & High \\
\hline 23 & 403.5 & 1.8 & 726.30 & High \\
\hline 24 & 380.5 & 0.8 & 304.40 & High \\
\hline 25 & 112.8 & 10.6 & 1195.68 & Very High \\
\hline 26 & 200.6 & 1.6 & 320.96 & High \\
\hline 27 & 231.0 & 3.0 & 693.00 & High \\
\hline 28 & 195.3 & 1.8 & 351.54 & High \\
\hline 29 & 423.1 & 1.1 & 465.41 & High \\
\hline 30 & 437.7 & 0.8 & 348.56 & High \\
\hline
\end{tabular}

\section{Conclusion}

An assessment of the protective capacity of the vadose zone overlying aquifer systems in kaltungo area was carried out using secondary geo-electric parameters computed from VES data generated from 30 points in the field. The parameters considered here are longitudinal conductance (S) and Transverse Resistance here synonymous with Transmissivity (T) computed based on established relation between geo-electric resistivity and aquifer parameters (Dar Zarouk Parameters) thus;

$\mathrm{S}=\mathrm{h} / \rho \mathrm{a}$

$\mathrm{T}=$ h. $\rho \mathrm{a}$

The results from the two parameters as presented in tables 4 and 5 revealed that the vadose zone (overburden materials) in the study area offer poor protection to the underlying aquifer systems. The study has confirmed that using geo-electric parameters can be useful in groundwater quality studies. The relation between electrical resistivity, layer thickness and aquifer properties has also been confirmed by this study hence combining geophysical resistivity methods and other groundwater quality vulnerability mapping can form a good basis for groundwater sustainability studies.

\section{Acknowledgement}

The Authors are grateful to the staff of Holocene Engineering Nigerian limited for providing equipment and man power for field work and the management of Gombe State University for sponsoring part of the data collection.

\section{Reference}

[1] Ademilua, O. L. and Ogungbemi,O. S. Evaluating aquifer protective capacity of ground water resources within AfeBabalola University, Ado-Ekiti. Transnational Journal of Science and Technology, 2013, 3(6): 1857-8047.

[2] Ahmad, S. De Marsily, G. Comparison of Geophysical methods for estimating transmissivity and specific capacity. Water Resour. Res., 1987, 23: 1717-1723.

Legend:

[3] Arabi, A. S., Nur, A., Dewu, B.B.M. Hydro Geo-Electrical Investigation in Gombe Town and Environs, Northeastern Nigeria. J. Appl. Sci. Environ. Management. 2009, 13(3): 65-68.

High to very High Transmissivity magnitude

[4] Batte, A.G., Barifaiyo, E. Kiberu, J.M., Kawule, W., Muwanga, A., Owor, M., Kisekulo J. Correlation of geo-electric data with aquifer parameters to delineate the groundwater potential of hard rock terrain in Central Uganda. Pure Appl Geophys. 2010, 167(12): 1549-1559.

Figure 12. Vadose Zone Transmissivity Map

[5] Benkhelil, M.J. The Evolution of the Cretaceous 
Benue trough, Nigeria. Journal of Africa earth Sciences, ,1989(8): 251-282.

[6] Dasagues, A. Modelling base flow from alluvial aquifer using hydraulic conductivity data obtained from a derived relation with apparent resistivity. J. Hydrogeol. 1997, 5: 97-108.

[7] Dhakate, R., Singh, V.S. Estimation of Hydraulic Parameters from surface Geophysical Methods, Kaliapani Ultramafic Complex, Orissa, India. journal of Environmental Hydrology, 2005, 13(12): 12.

[8] Dike, E.F.C., Shemang, E.M., Dan Hassan, M.A. Water exploration in ATBU main Campus at Gubi using electromagnetic and resistivity techniques. unpublished report, 1994: 1-13.

[9] Egbai, J.C. Geo-electrical sounding for determination of aquifer transmissivity. Aust. J.Basic Appl. Sci. 2011, 5 (6): 1209-1214.

[10] Ezeh, C.C. Geo-electrical Studies for estimating aquifer Hydraulic properties in Enugu State Nig. Int. J. Phys. Sci., 2011, 6(14): 3319-3329.

[11] Frohlich, R.K., Fisher, J.J., Summerly, E. Electric Hydraulic conductivity correlation in fractured crystalline bedrock Central landfill Rhode Island USA. J.Appl.Geophysics, 1996, 35: 249-529.

[12] Henriet, J.P. Direct applications of the Dar Zarrouk parameters in ground water surveys. Geophy.Prospect., 1976, 24: 345-353.

[13] Kelly, W.E. Geo-electric Sounding for estimating aquifer hydraulic conductivity. Groundwater, 1977, 15: 420-424.

[14] Kirsch R. Groundwater Geophysics- A tool for hydrogeology. Springer- Verlag, Berlin Herdelberg, 2006: 493.

[15] Maillet, R. The fundamental equations of electrical prospecting.Geophysics. Journal of Hydrology, 1947, 50: 393-399. http://dx.doi.org/10.1016/0022.

[16] Mbonu,P.D.C., Ebeniro, J.C., Ofoegbu, C.O., Ekine, A.S. Geoelectric Sounding for the Determination of Aquifer Characteristics in parts of the Umahia Area of Nigeria. Geophysics, 1991, 56(2): 284-291.

[17] Mboringong, M.N., Ashano E.C., Olasehinde, A., Tabale, R.P., Abubakar Y. Evaluation of arsenic in rocks of Kaltungo area. journal of natural sciences research. 2013, 3(4): 25-30.

[18] Mundel, J.A., Lother, L., Oliver, E.M., Allen L.S. Aquifer Vulnerability analysis for water resource production" Indian Department of Environmental Management, 2003: 25.

[19] Niwas, S., Singhal, D.C. Estimation of Aquifer Transmissivity from Dar Zarouk Parameters in porous Media. Journal of Hydrology, 1988, 50: 393399.

[20] Oladapo, M.I., Mohammed,M.Z., Adeoye, O.O., Adetola, B.A. Geoelectrical investigation of the Ondo State Housing Corporation Estate Ijapo Akure, Southwestern Nigeria. Res. J. Appl. Sci. Eng. Technol., 2004, 2(8): 785-791,

[21] Oladapo, M., Akintorinwa, O. Hydrogeophysical study of Ogbese South Western Nigeria. Global journal of pure and applied sciences, 2007, 13: 55-61.

[22] Olorunfemi, M. and okhue, E. Hydrogeologic and geologic significance of a Geoelectric Survey at IleIfe, Nigeria. Journal of mining and geology, 1992, 28: 221-229.

[23] Onuoha, K.M., Mbazi, F.G.C. Aquifer transmissivity from electrical sounding data,the case of Ajali Sandstone aquifer south West of Enugu, Nigeria in Ofoegbu, C.O., Ed Groundwater and minerl resources of Nigeria. Vieweg-Verlag, 1988: 17-30.

[24] Ponzini, G., Ostroman, A., Mollinai, M. Empirical relation between electrical transverse resistance and hydraulic transmissivity. Geo-exploration, 1984, 22: $1-15$.

[25] Sa'ad, A.S., Baba, S. Geology and Petrographic study of the Granitic rocks of Kaltungo Inlier, Northeastern Nigeria. Journal of Applied Geology and Geophysics, 2017, 5 (1): 75-83

[26] Utom, A., U., Odoh, B. I., Okoro, A.U. Estimation of Aquifer Transmissivity using Dar Zarouk Parameters from Surface Resistivity Measurements: A Case History from Parts of Enugu Town Nigeria. Journal of Water Resources and Protection, 2014(4): 993-1000.

[27] Zaborski P. M. Guide to the Cretaceous System in the upper part of the Upper Benue Trough, Northeastern Nigeria. Afr. Geosci. Rev., 1973, 10(1\&2): 13-32. 